\title{
Protein segregase meddles in remodeling of mRNA-protein complexes
}

\author{
Chyi-Ying A. Chen and Ann-Bin Shyu' \\ Department of Biochemistry and Molecular Biology, The University of Texas Medical School at Houston, Houston, Texas 77030, USA
}

\begin{abstract}
Remodeling of RNA-protein complexes (mRNPs) plays a critical role in mRNA biogenesis and metabolism. However, relatively little is known about the underlying mechanism and regulation of the mRNP remodeling. In this issue of Genes \& Development, Zhou and colleagues (pp. 1046-1058) report that a protein remodeling machine, the p97-UBXD8 complex, disassembles mRNPs containing the AU-rich elements (AREs) bound by HuR proteins in a nondegradative, ubiquitin signaling-dependent manner, revealing a novel mechanism to regulate mRNA turnover.
\end{abstract}

Messenger RNAs (mRNAs) associate with a wide variety of proteins during mRNA biogenesis and metabolism. The resulting mRNA-protein complexes (mRNPs) undergo a series of remodeling events that are affected by and/or influence pre-mRNA processing, nuclear export, translation, localization, and the mRNA decay machinery (Moore 2005; Shyu et al. 2008). Assembly and disassembly of mRNPs are highly dynamic processes. A wellknown example that illustrates the dynamic nature and the importance of mRNP remodeling is the varying array of AU-rich element-binding proteins (ARE-BP) found to be associated with the cognate transcripts during cellular stress responses and the corresponding alterations in the stability of the transcripts (Stoecklin and Anderson 2007; Clement et al. 2011). Even though the fundamental importance of mRNP remodeling has come to be recognized over the past decade, relatively little is known about the underlying mechanisms and regulation of the remodeling. Much of our current knowledge about mRNP remodeling comes from studies of ATP-dependent RNA helicases (e.g., see Tran et al. 2007; Gehring et al. 2009; Franks et al. 2010). The unwinding of structured mRNAs by these helicases helps to modify the protein composition of mRNPs during each step of mRNA metabolism (Linder and Jankowsky 2011). For instance, RNA helicase action changes the RNA secondary or tertiary structure during pre-mRNA splicing, leading to exchange of snRNPs (Cordin and Beggs 2013). However,

[Keywords: HuR; mRNA stability; ubiquitin]

${ }^{1}$ Corresponding author

E-mail ann-bin.shyu@uth.tmc.edu

Article is online at http://www.genesdev.org/cgi/doi/10.1101/gad.219469.113. it remains unclear as to what other factors may contribute to the protein unfolding presumed to occur during "extraction" of proteins from an mRNP, as an RNA helicase is not known for having such an activity. Moreover, AREs rarely adopt any secondary structure; therefore, it is unclear as to whether an RNA helicase is involved in the disassociation of ARE-BPs from the mRNP.

There are now several instances where the exchange of a destabilizing ARE-BP (such as TTP or KSRP) in favor of a stabilizing ARE-BP (predominantly HuR, a ubiquitously expressed RNA-binding protein [RBP]) accounts for the stabilization of an ARE-containing mammalian mRNA in response to environmental or physiological stimuli (Fig. 1A,B; e.g., see Brennan et al. 2000; Lal et al. 2004; Hinman and Lou 2008). HuR is one of the beststudied factors that recognize various AREs present in the 3' untranslated regions (UTRs) of transcripts coding for cytokines, proto-oncogenes, and transcription factors (Chen and Shyu 1995; Barreau et al. 2006). HuR's affinity for its ARE targets appears to be one of the highest among ARE-BPs (Ma et al. 1996; Chen et al. 2002; Lal et al. 2004). Thus, it is plausible that HuR simply outcompetes destabilizing ARE-BPs for occupation of AREs on target mRNAs, consequently stabilizing an otherwise unstable message (Srikantan and Gorospe 2012). The list of mRNAs found to be regulated by HuR is steadily growing, along with appreciation of the functional importance of post-transcriptional gene regulation by HuR in human physiology and pathology (von Roretz et al. 2011; Srikantan and Gorospe 2012). In addition to its original role as an mRNA-stabilizing factor, several studies have demonstrated that HuR is a multitasking protein involved in almost every aspect of mRNA processing. Overexpression of HuR is linked to various pathologies in which increased stabilization and/or translation of certain key mRNAs causes undesirably prolonged cell responses (Abdelmohsen and Gorospe 2010; Srikantan and Gorospe 2012). As a result, HuR has been attributed a fundamental role in immunopathology, inflammation, and cancer (e.g., see Dixon et al. 2001; Katsanou et al. 2005; Yiakouvaki et al. 2012).

Given that HuR is one of the most abundant and ubiquitously expressed proteins in mammalian cells and the high affinity of HuR for ARE targets, an intriguing and important question is how HuR might be "pulled out" from an mRNP once cellular conditions call for the 

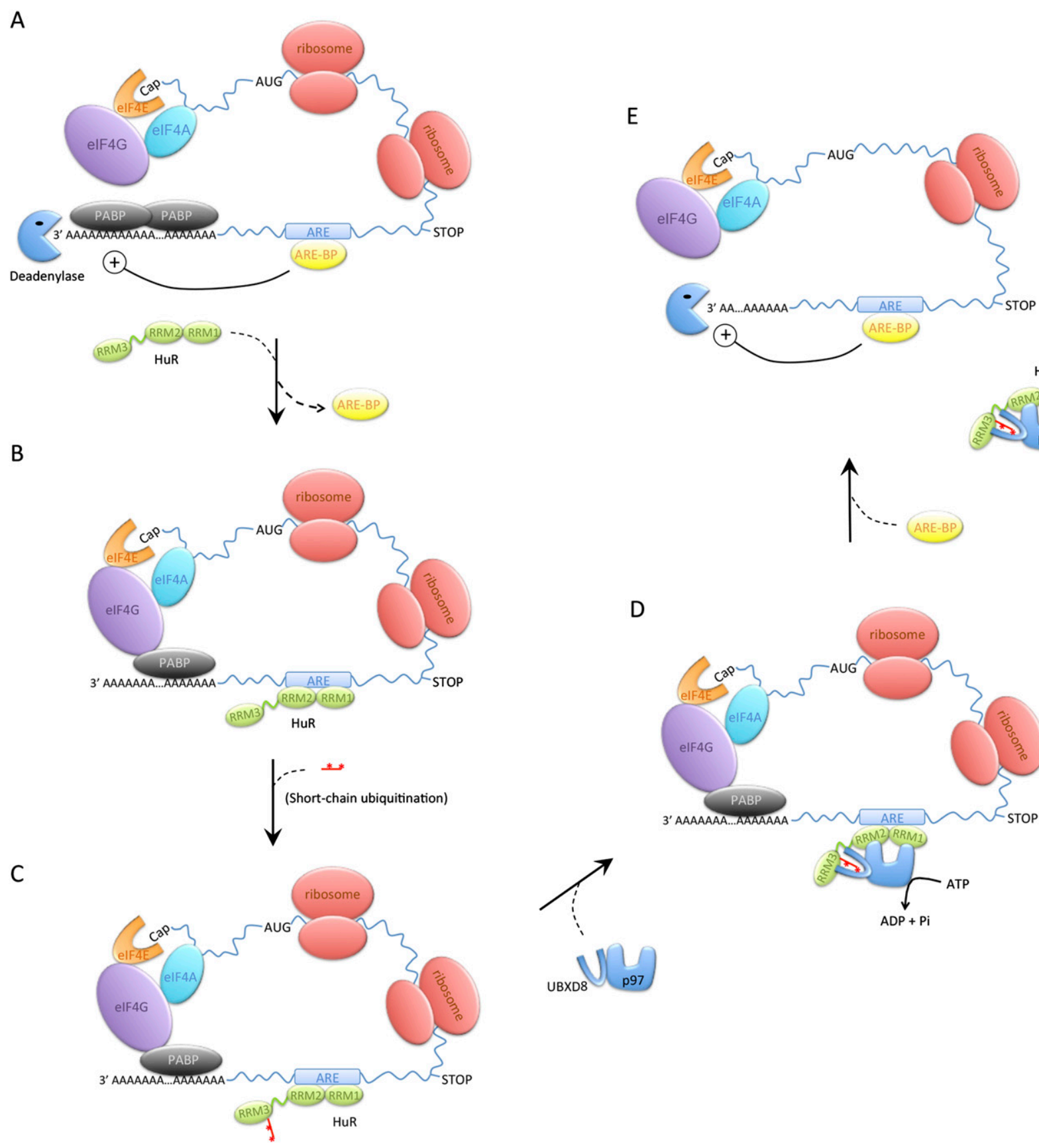

HuR
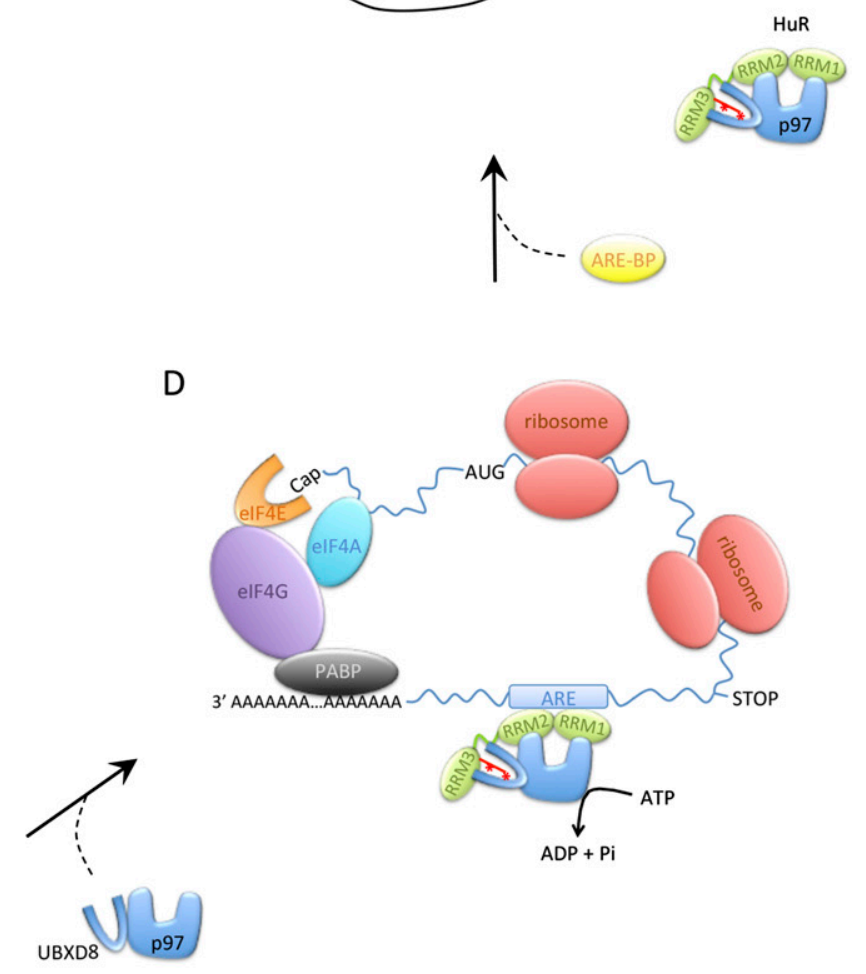

Figure 1. A model showing how nondegradative ubiquitin signaling leads to remodeling of HuR-associated mRNPs and alteration of the mRNA stability. See the text for details. (Yellow oval) Destabilizing ARE-BP.

instability of the mRNA to be restored by association with a destabilizing ARE-BP.

Recently, there have been significant advances in understanding how post-translational modifications of HuRmainly phosphorylation-affect its subcellular localization and stability (Kim et al. 2008a; Eberhardt et al. 2012). It appears that HuR's association with its target mRNAs in the cytoplasm is controlled by decreases in its cytoplasmic concentration through either degrading the protein or moving it to the nucleus. Thus, phosphorylation of HuR plays a role in controlling the protein's abundance in the cytoplasm (Eberhardt et al. 2012). While these scenarios can explain how destabilizing ARE-BPs regain the opportunity to associate with AREcontaining mRNAs when HuR levels drop, they do not readily explain how an exchange between HuR and other ARE-BPs can happen under conditions where the cytoplasmic HuR concentration remains high following a stimulus.

In this issue of Genes \& Development, Zhou et al. (2013) report on a nondegradative, ubiquitin signalingdependent disassembly of $\mathrm{mRNPs}$ promoted by a protein 
remodeling machine, the p97-UBXD8 complex. This represents a novel mechanism to regulate the stability of HuR targeted, ARE-containing mRNAs. In an effort to identify HuR-interacting proteins in HeLa cells, the investigators first noticed the RNA-independent interaction of HuR with p97 and one of its cofactors, UBXD8. p97 is a member of the AAA (ATPases associated with diverse cellular activities) family of proteins and is considered a protein remodeling machine (Meyer et al. 2012). Typically, p97 recognizes a ubiquitinated substrate protein through one of its cofactors and then uses the energy from ATP hydrolysis to remove the ubiquitinated protein from a complex with other proteins (Meyer et al. 2012); this action of p97 is also known as segregase activity (Ramadan et al. 2007; Acs et al. 2011). An important feature of the action of the p97-cofactor complex that caught the investigators' attention is that the extracted ubiquitinated proteins are not necessarily degraded by the ubiquitin-proteasome system (Meerang et al. 2011). Rather, the proteins can be deubiquitinated and recycled for subsequent use through what is termed the nondegradative pathway. Using a coimmunoprecipitation approach, Zhou et al. (2013) showed that HuR does not interact with either p97 or UBXD8 alone but instead with the p97-UBXD8 complex. Moreover, knocking down either p97 or UBXD8 in HeLa cells led to specific stabilization of an HuR target mRNA, p21 (Wang et al. 2000), whereas depleting HuR resulted in destabilization of the p21 mRNA. The investigators further used two complementary experiments to show that the regulation of p21 mRNA by p97 and UBXD8 depends on HuR and its target sequence. First, when the ARE was removed from the 3' UTR of the p21 mRNA sequence carried by a reporter, knocking down p97 or UBXD8 no longer affected the reporter's expression level. Second, the increase in p21 mRNA level upon depleting the p97/UBXD8 complex was significantly attenuated when HuR was also knocked down. Consistent with a general role of the p97-UBXD8 complex in mRNA stability control, the investigators obtained a similar effect on two additional HuR target mRNAs: MKP-1 and SIRT1 (Abdelmohsen et al. 2007; Kuwano et al. 2008)

Considering the ability of p97-UBXD8 to recognize ubiquitinated proteins and remodel protein complexes where the ubiquitinated targets reside (Schuberth and Buchberger 2008; Meyer et al. 2012), the investigators asked whether the HuR protein could be ubiquitinated and subsequently ejected from ARE-containing mRNPs. They found that immunoprecipitated HuR exhibits a range of ubiquitination, with a predominant $\sim 50-\mathrm{kDa}$ species corresponding to the addition of two ubiquitin units. Using an array of ubiquitin mutants, each mutated at a specific lysine $(\mathrm{K})$ residue, the investigators pinpointed K29 of ubiquitin as being required for generation of the 50 -kDa ubiquitinated species. The investigators further showed that short chain ubiquitination at K29 is not a proteolytic signal, in clear distinction from the effect of ubiquitination at K48 (Pickart 1997). Instead, a K29linked ubiquitin chain on HuR allows UBXD8 to interact specifically with HuR. This unexpected and exciting finding may offer an opportunity for future investigation of the role of this particular ubiquitin linkage, which remains largely unexplored (Russell and Wilkinson 2004; Chastagner et al. 2006). In reciprocal experiments, using a series of deletion and point mutations of HuR and an in vitro ubiquitination assay, the investigators identified and confirmed that the two lysine residues (K313 and K326) in the third RRM of HuR are the ones subject to K29-linked ubiquitination. Lysine-to-arginine changes at these two positions did not affect HuR's subcellular distribution, RNA binding, or stability but rather diminished HuR's interaction with UBXD8. This suggests that K29-linked ubiquitination of HuR strengthens its interaction with the p97-UBXD8 complex.

The next question is whether the addition of a K29linked ubiquitin chain to HuR at K313 and K326 has any effect on HuR's role in stabilizing its target mRNAs. If yes, then how? In light of previous findings that p97-UBX extracts target proteins from protein complexes (Meyer et al. 2012), the investigators hypothesized that p97UBXD8 alters HuR-dependent mRNA stability control by acting on an mRNP complex to release HuR. In testing this hypothesis, the investigators first showed that depleting p97 or UBXD8 increased HuR association with its target transcript, p21 mRNA, whereas increasing p97 levels decreased the association. The key experiment involved a well-designed, in vitro mRNP disassembly assay. The investigators used a biotinylated RNA bearing the p21 ARE sequence as bait to capture HuR or ubiquitinated HuR proteins from lysates from cells ectopically expressing myc-HuR and HA-ubiquitin. They then mixed recombinant p97/UBXD8 proteins and ATP with the HuR/p21 RNP complex, stopping the reaction at different time points over $60 \mathrm{~min}$ to evaluate HuR levels in the supernatant (released) versus in the precipitate (bound). The results revealed a progressive, ATP-dependent release of ubiquitinated HuR from the mRNP complex. This release of HuR from p21 ARE was not observed when mutant HuR (K313/326) or mutant ubiquitin (K29R) was tested. In a reciprocal experiment, the investigators observed an increase in p21 mRNA dissociation from HuR when the p97/UBXD8 complex and ATP were present. Collectively, these results strongly support the proposed regulatory scenario. Ubiquitination of HuR at K313/326 promotes strong association with the p97-UBXD complex (Fig. 1C,D). Then, ATP hydrolysis by p97 allows the p97-UBXD8 complex to induce a conformational change in HuR, leading to its release from the ARE and its subsequent replacement by an ARE-BP that destabilizes the mRNA (Fig. 1D,E).

Zhou et al. (2013) take the story further still. Mindful that HuR plays a critical role in modulating cytoplasmic mRNA abundance during various cellular stress responses (Gallouzi et al. 2000; Wang et al. 2000; Hinman and Lou 2008; Srikantan and Gorospe 2012), the investigators found that ubiquitination of HuR was decreased upon UV irradiation or heat shock. These stressors also increased association of HuR with p21 and increased the steady-state abundance of the p $21 \mathrm{mRNA}$. These results suggest a physiological link between stress responses 
and p97-UBXD8-mediated remodeling of HuR-mRNPs. Phosphorylation of HuR is critical for the HuR-mediated stress response (Abdelmohsen et al. 2007; Kim et al. 2008b); thus, as Zhou et al. (2013) pointed out, it will be interesting to investigate possible connections between the phosphorylation and nondegradative ubiquitination signaling pathways during regulation of $\mathrm{HuR}$ in response to a specific stimulus. A related further question is whether phosphorylation at one or more specific HuR sites is required for the nondegradative ubiquitination of HuR.

A couple of key issues remain unresolved by the Zhou et al. (2013) study. First, UBXD8 associates with the endoplasmic reticulum (ER) and lipid droplets through a transmembrane domain, and UBXD8 is also linked to ER-associated protein degradation and lipid metabolism (Zehmer et al. 2009; Lee et al. 2010). It is thus unclear exactly how UBXD8 can function in the cytosol to remodel an array of HuR-associated mRNAs. Another issue is that dissociation of HuR from the ARE sequence is supported by assays with proteins pulled down from complex cell extracts, so the p97-UBXD8 complex could potentially include a number of other proteins that are required for the segregase activity that results in HuR ejection. Thus, it remains possible that the release of HuR from the RNA could be an indirect process involving other unknown factors. An in vitro assay using purified recombinant proteins may help address this issue.

Given that the p97-UBXD8 complex can interact with a number of other RBPs besides HuR, it will be interesting to learn whether compromising the mRNP remodeling function of p97/UBXD8 affects the fate of cytoplasmic mRNAs in general in terms of their stability, translation, or localization. Moreover, other RBPs may recruit p97UBXD8 for remodeling other mRNPs. p97 can associate with an array of distinct cofactors, so one could imagine that other RBPs associate with different p97-cofactor complexes. Thus, the discovery of the p97-UBXD8 machine's role in HuR-containing mRNP remodeling may simply represent the tip of the iceberg, with broader implications for general mRNP remodeling than are revealed by this study. Nondegradative effects of protein ubiquitination are emerging as exciting aspects of ubiquitin biology, but only a few examples have been reported thus far (Chen and Sun 2009). The present study by Zhou et al. (2013) may open up a new direction for investigating the mechanisms and functions of nondegradative ubiquitin signaling in mRNP remodeling. Mutations in p97 have been genetically linked with inclusion body myopathy, Paget's disease, frontotemporal dementia, and amyotrophic lateral sclerosis (Kimonis et al. 2008; Johnson et al. 2010; Tresse et al. 2010), yet the molecular pathogeneses of these diseases are not fully understood. It is conceivable that the severe neurodegenerative diseases associated with p97 mutations involve a defect in the pathway brought to light by the study by Zhou et al. (2013).

\section{Acknowledgments}

The work by the laboratory of A.-B.S. is supported by grants from the National Institutes of Health and by the Houston Endowment, Inc.

\section{References}

Abdelmohsen K, Gorospe M. 2010. Posttranscriptional regulation of cancer traits by HuR. Wiley Interdiscip Rev RNA 1: 214-229.

Abdelmohsen K, Pullmann R Jr, Lal A, Kim HH, Galban S, Yang X, Blethrow JD, Walker M, Shubert J, Gillespie DA, et al. 2007. Phosphorylation of HuR by Chk2 regulates SIRT1 expression. Mol Cell 25: 543-557.

Acs K, Luijsterburg MS, Ackermann L, Salomons FA, Hoppe T, Dantuma NP. 2011. The AAA-ATPase VCP/p97 promotes 53BP1 recruitment by removing L3MBTL1 from DNA double-strand breaks. Nat Struct Mol Biol 18: 1345-1350.

Barreau C, Paillard L, Osborne HB. 2006. AU-rich elements and associated factors: Are there unifying principles? Nucleic Acids Res 33: 7138-7150.

Brennan CM, Gallouzi IE, Steitz JA. 2000. Protein ligands to HuR modulate its interaction with target mRNAs in vivo. J Cell Biol 151: 1-14.

Chastagner P, Israel A, Brou C. 2006. Itch/AIP4 mediates Deltex degradation through the formation of K29-linked polyubiquitin chains. EMBO Rep 7: 1147-1153.

Chen AC-Y, Shyu A-B. 1995. AU-rich elements: Characterization and importance in mRNA degradation. Trends Biochem Sci 20: 465-470.

Chen ZJ, Sun LJ. 2009. Nonproteolytic functions of ubiquitin in cell signaling. Mol Cell 33: 275-286.

Chen C-YA, Xu N, Shyu A-B. 2002. Highly selective actions of HuR in antagonizing AU-rich element-mediated mRNA destabilization. Mol Cell Biol 22: 7268-7278.

Clement SL, Scheckel C, Stoecklin G, Lykke-Andersen J. 2011. Phosphorylation of tristetraprolin by MK2 impairs AU-rich element mRNA decay by preventing deadenylase recruitment. Mol Cell Biol 31: 256-266.

Cordin O, Beggs JD. 2013. RNA helicases in splicing. RNA Biol 10: $83-95$.

Dixon DA, Tolley ND, King PH, Nabors LB, McIntyre TM, Zimmerman GA, Prescott SM. 2001. Altered expression of the mRNA stability factor HuR promotes cyclooxygenase-2 expression in colon cancer cells. J Clin Invest 108: 1657-1665.

Eberhardt W, Doller A, Pfeilschifter J. 2012. Regulation of the mRNA-binding protein HuR by posttranslational modification: Spotlight on phosphorylation. Curr Protein Pept Sci 13: 380-390.

Franks TM, Singh G, Lykke-Andersen J. 2010. Upf1 ATPasedependent mRNP disassembly is required for completion of nonsense-mediated mRNA decay. Cell 143: 938-950.

Gallouzi IE, Brennan CM, Stenberg MG, Swanson MS, Eversole A, Maizels N, Steitz JA. 2000. HuR binding to cytoplasmic mRNA is perturbed by heat shock. Proc Natl Acad Sci 97: 3073-3078.

Gehring NH, Lamprinaki S, Kulozik AE, Hentze MW. 2009. Disassembly of exon junction complexes by PYM. Cell 137: 536-548.

Hinman M, Lou H. 2008. Diverse molecular functions of $\mathrm{Hu}$ proteins. Cell Mol Life Sci 65: 3168-3181.

Johnson JO, Mandrioli J, Benatar M, Abramzon Y, Van Deerlin VM, Trojanowski JQ, Gibbs JR, Brunetti M, Gronka S, Wuu J, et al. 2010. Exome sequencing reveals VCP mutations as a cause of familial ALS. Neuron 68: 857-864.

Katsanou V, Papadaki O, Milatos S, Blackshear PJ, Anderson P, Kollias G, Kontoyiannis DL. 2005. HuR as a negative posttranscriptional modulator in inflammation. Mol Cell 19: 777-789.

Kim HH, Yang X, Kuwano Y, Gorospe M. 2008a. Modification at $\mathrm{HuR}(\mathrm{S} 242)$ alters HuR localization and proliferative influence. Cell Cycle 7: 3371-3377. 
Kim HH, Abdelmohsen K, Lal A, Pullmann R Jr, Yang X, Galban S, Srikantan S, Martindale JL, Blethrow J, Shokat KM, et al. 2008b. Nuclear HuR accumulation through phosphorylation by Cdk1. Genes Dev 22: 1804-1815.

Kimonis VE, Fulchiero E, Vesa J, Watts G. 2008. VCP disease associated with myopathy, Paget disease of bone and frontotemporal dementia: Review of a unique disorder. Biochim Biophys Acta 1782: 744-748.

Kuwano Y, Kim HH, Abdelmohsen K, Pullmann R, Martindale JL, Yang X, Gorospe M. 2008. MKP-1 mRNA stabilization and translational control by RNA-binding proteins HuR and NF90. Mol Cell Biol 28: 4562-4575.

Lal A, Mazan-Mamczarz K, Kawai T, Yang X, Martindale JL, Gorospe M. 2004. Concurrent versus individual binding of HuR and AUF1 to common labile target mRNAs. EMBO $J$ 23: 3092-3102.

Lee JN, Kim H, Yao H, Chen Y, Weng K, Ye J. 2010. Identification of Ubxd8 protein as a sensor for unsaturated fatty acids and regulator of triglyceride synthesis. Proc Natl Acad Sci 107: 21424-21429.

Linder P, Jankowsky E. 2011. From unwinding to clamping-the DEAD box RNA helicase family. Nat Rev Mol Cell Biol 12: 505-516.

Ma WJ, Cheng S, Campbell C, Wright A, Furneaux H. 1996. Cloning and characterization of HuR, a ubiquitously expressed Elav-like protein. J Biol Chem 271: 8144-8151.

Meerang M, Ritz D, Paliwal S, Garajova Z, Bosshard M, Mailand N, Janscak P, Hubscher U, Meyer H, Ramadan K. 2011. The ubiquitin-selective segregase $\mathrm{VCP} / \mathrm{p} 97$ orchestrates the response to DNA double-strand breaks. Nat Cell Biol 13: 1376-1382.

Meyer H, Bug M, Bremer S. 2012. Emerging functions of the VCP/p97 AAA-ATPase in the ubiquitin system. Nat Cell Biol 14: 117-123.

Moore MJ. 2005. From birth to death: The complex lives of eukaryotic mRNAs. Science 309: 1514-1518.

Pickart CM. 1997. Targeting of substrates to the 26S proteasome. FASEB J 11: 1055-1066.

Ramadan K, Bruderer R, Spiga FM, Popp O, Baur T, Gotta M, Meyer HH. 2007. Cdc48/p97 promotes reformation of the nucleus by extracting the kinase Aurora B from chromatin. Nature 450: 1258-1262.

Russell NS, Wilkinson KD. 2004. Identification of a novel 29linked polyubiquitin binding protein, Ufd3, using polyubiquitin chain analogues. Biochemistry 43: 4844-4854.

Schuberth C, Buchberger A. 2008. UBX domain proteins: Major regulators of the AAA ATPase Cdc48/p97. Cell Mol Life Sci 65: 2360-2371.

Shyu AB, Wilkinson MF, van Hoof A. 2008. Messenger RNA regulation: To translate or to degrade. EMBO J 27: 471-481.

Srikantan S, Gorospe M. 2012. HuR function in disease. Front Biosci 17: 189-205.

Stoecklin G, Anderson P. 2007. In a tight spot: ARE-mRNAs at processing bodies. Genes Dev 21: 627-631.

Tran EJ, Zhou Y, Corbett AH, Wente SR. 2007. The DEAD-box protein Dbp5 controls mRNA export by triggering specific RNA:protein remodeling events. Mol Cell 28: 850859.

Tresse E, Salomons FA, Vesa J, Bott LC, Kimonis V, Yao T-P, Dantuma NP, Taylor JP. 2010. VCP/p97 is essential for maturation of ubiquitin-containing autophagosomes and this function is impaired by mutations that cause IBMPFD. Autophagy 6: 217-227.

von Roretz C, Marco SD, Mazroui R, Gallouzi I-E. 2011. Turnover of AU-rich-containing mRNAs during stress: A matter of survival. Wiley Interdiscip Rev RNA 2: 336-347.
Wang W, Furneaux H, Cheng H, Caldwell MC, Hutter D, Liu Y, Holbrook N, Gorospe M. 2000. HuR regulates p21 mRNA stabilization by UV light. Mol Cell Biol 20: 760-769.

Yiakouvaki A, Dimitriou M, Karakasiliotis I, Eftychi C, Theocharis S, Kontoyiannis DL. 2012. Myeloid cell expression of the RNA-binding protein HuR protects mice from pathologic inflammation and colorectal carcinogenesis. J Clin Invest 122: 48-61.

Zehmer JK, Bartz R, Bisel B, Liu P, Seemann J, Anderson RGW. 2009. Targeting sequences of UBXD8 and AAM-B reveal that the ER has a direct role in the emergence and regression of lipid droplets. J Cell Sci 122: 3694-3702.

Zhou HL, Geng C, Luo G, Lou H. 2013. The p97-UBXD8 complex destabilizes mRNA by promoting release of ubiquitinated HuR from mRNP. Genes Dev (this issue). doi: 10.1101/ gad.215681.113. 


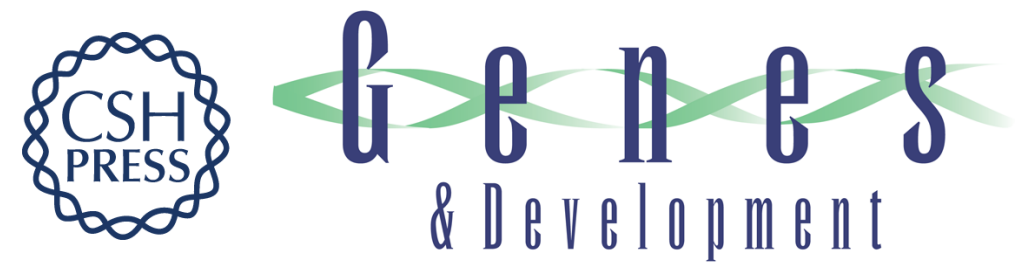

\section{Protein segregase meddles in remodeling of mRNA-protein complexes}

Chyi-Ying A. Chen and Ann-Bin Shyu

Genes Dev. 2013, 27:

Access the most recent version at doi:10.1101/gad.219469.113

\section{Related Content The p97UBXD8 complex destabilizes mRNA by promoting release of ubiquitinated HuR from mRNP \\ Hua-Lin Zhou, Cuiyu Geng, Guangbin Luo, et al. \\ Genes Dev. May , 2013 27: 1046-1058 \\ References This article cites 43 articles, 12 of which can be accessed free at: \\ http://genesdev.cshlp.org/content/27/9/980.full.html\#ref-list-1 \\ Articles cited in: \\ http://genesdev.cshlp.org/content/27/9/980.full.html\#related-urls \\ License \\ Email Alerting \\ Service \\ Receive free email alerts when new articles cite this article - sign up in the box at the top right corner of the article or click here.}

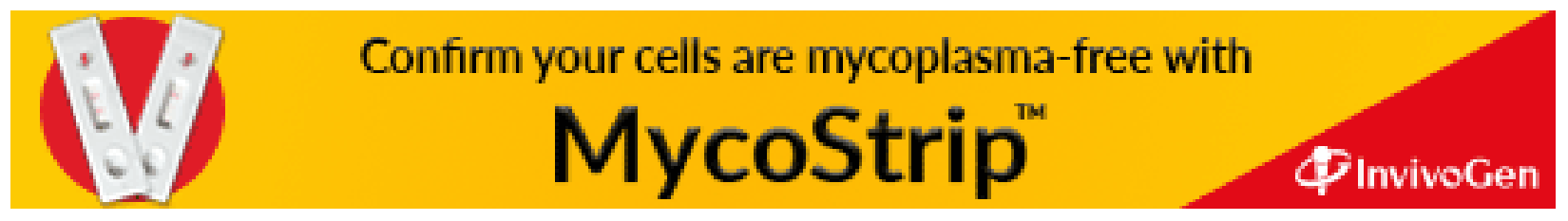

